$\mathbf{S}_{c}^{\mathrm{e}}$ da a conocer un nuevo registro de Cyathea fulva (M. Martens \& Galeotti) Fée, para la flora del Estado de México. Este taxón se encontró como parte de los estudios sobre ecología y conservación de la biodiversidad en la Sierra Nanchititla, ubicada al suroeste del Estado de México. Se describe su posición taxonómica, el hábitat donde se encontró la población, así como su distribución a nivel mundial y nacional. Finalmente, se comentan algunas amenazas presentes en la Sierra Nanchititla que pueden afectar directamente a la población de $C$. fulva.

Con el fin de corroborar el nuevo registro se revisó el material depositado en los Herbarios MEXU, ENCB, XAL, IEB, CHAPA y FCME, se consultó la información de las colecciones conectadas en línea en la Red Mundial de Información sobre Biodiversidad (REMIB) y la bibliografía especializada sobre el tema, como el trabajo de Matuda (1956), Tejero-Díez y Arreguín-Sánchez (2004) y Mickel y Smith (2004).

Mickel y Smith (2004) indicaron la presencia de Alsophila firma (Baker) D. S. Conant, también de la familia Cyatheaceae, en los alrededores de Tejupilco, un área relativamente cercana a la localidad aquí citada (40 km por carretera), con base en material recolectado por G. B. Hinton y depositado en el Herbario Nacional de los Estados Unidos (US). De esta forma serían dos las especies de helechos arborescentes en la entidad, sin embargo, dado que A. firma no ha sido colectada nuevamente en la región, su presencia es dudosa.

La Sierra Nanchititla se localiza en el suroeste del Estado de México, en los límites con los Estados de Michoacán y Guerrero, en el municipio de Luvianos, entre las coordenadas $100^{\circ}$ 36 ' 49" y $100^{\circ} 16^{\prime} 03$ " longitud O y $18^{\circ} 45^{\prime} 13$ " y $19^{\circ} 04^{\prime} 22^{\prime \prime}$ latitud N. Forma parte de la región fisiográfica Cuenca del Río Las Balsas. Posee una

\title{
Primer ReGistro de Cyathea Fulva (Cyatheaceae, Polypodiopsida) en EL ESTADO DE MÉxICO
}

\author{
Carmen Zepeda ${ }^{1}$, Octavio Monroy-Vilchis ${ }^{2}$, \\ ERnesto Velázquez-Montes ${ }^{3,4}$ y Clarita RodríGuez-Soto ${ }^{2}$ \\ ${ }^{1}$ Centro de Investigación en Recursos Bióticos, Facultad de Ciencias \\ ${ }^{2}$ Estación Biológica Sierra Nanchititla, Facultad de Ciencias \\ Universidad Autónoma del Estado de México. Instituto Literario No. 100, \\ Col. Centro. C. P. 50000 Toluca, Estado de México, México. \\ ${ }^{3}$ Departamento de Biología Comparada, Facultad de Ciencias, \\ Universidad Nacional Autónoma de México, Ap. Postal 70-282, \\ Ciudad Universitaria, C. P. 04510, México, D. F. \\ ${ }^{4}$ Autor para correspondencia. Correo-e: vme@correo.unam.mx
}

topografía abrupta, con condiciones geomorfológicas y edáficas muy variadas y un intervalo altitudinal que va de los 420 a los 2100 m.s.n.m. Fue decretada Área Natural Protegida en 1977 (Aguilera, 1993, Anónimo, 1997) debido a su importancia biológica. En esta región se ha mencionado la presencia de algunas especies de fauna en peligro de extinción como es el caso de $\mathrm{He}$ loderma horridum (Wiegmann) (Monroy-Vilchis et al., 2005), Leopardus wiedii (Thomas) (Sánchez et al., 2002) y Panthera onca (Linnaeus) (MonroyVilchis et al., en prensa). Posee zonas relativamente bien conservadas de vegetación, destacando los bosques de Pinus y Quercus en las partes elevadas, el bosque tropical caducifolio en las bajas y en algunas laderas y cañadas se desarrolla el bosque en galería con algunos elementos de bosque mesófilo de montaña (figura 1).

\section{Ubicación taxonómica y biodiversidad}

Los helechos se ubican actualmente en el grupo llamado Monilofitas, que incluye entre otros a los helechos arborescentes, ubicados en la clase Polypodiopsida (Smith et al., 2006; Judd et al., 2007). La familia Cyatheaceae, que incluye únicamente a los helechos arborescentes escamosos, está representada por los géneros Alsophila R. Br., Cyathea J. E. Sm., Gymnosphaera Blume, Hymenophyllopsis K. I. Goebel y Sphaeropteris Bernh., que en conjunto contienen alrededor de 600 especies en todo el mundo (Smith et al., 2006). El género Cyathea se caracteriza porque las escamas de la base del pecíolo no tienen dientes oscuros en los márgenes ni en el ápice, sus márgenes están claramente diferenciados y la base del pecíolo no tiene espinas o si las tiene no son negras. Está compuesto por alrededor de 115 especies a nivel mundial, ocho de las cuales se encuentran en México (Mickel y Smith, 2004).

Cyathea fulva se distingue de las otras especies del género porque sus escamas, tanto del ápice del tallo como de la base del pecíolo, tienen células marginales diferentes en tamaño, forma y orientación a las de las células centrales, presenta indusios globosos, tricomas y escamas buladas de color pardo pálido en las venas medias del lado abaxial de los últimos segmentos y las pínnulas tienen pedicelos entre 1 


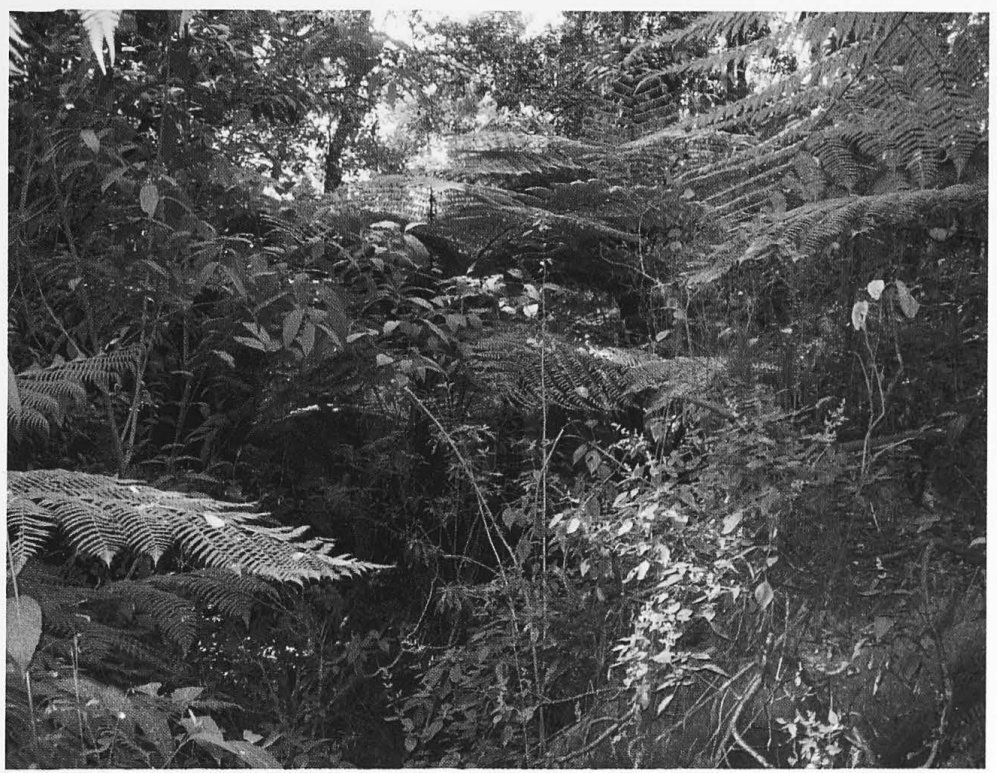

Figura 1. Población de Cyathea fulva en la cañada Río Frío.

y $2 \mathrm{~mm}$ de largo; todas estas características concuerdan con el material recolectado en la zona (figura 2). El taxón más cercano a $C$. fulva es $C$. divergens Kunze var. tuerckheimii (Maxon) R.M. Tryon que se distingue porque carece de tricomas, sus escamas son pardo oscuras, generalmente planas y se concentran principalmente en las axilas de las pinnas, además sus pínnulas tienen pedicelos entre 1 a $6 \mathrm{~mm}$ de largo (Mickel y Smith, 2004).

La distribución geográfica de $C$. fulva va desde México hasta Ecuador, incluyendo Guatemala, Honduras, El Salvador, Nicaragua, Costa Rica, Panamá, Venezuela y Colombia; en México se encuentra en Chiapas, Guerrero, Hidalgo, Oaxaca, Puebla y Veracruz (Mickel y Smith, 2004), de tal forma que su área de distribución se amplía con este hallazgo para el Estado de México. Era muy probable la presencia de dicha especie en la entidad, ya que Lorea-Hernández y VelázquezMontes (1998) la citan para la Sierra Madre del Sur en Guerrero y Fernández-Nava et al. (1998) en la cuenca del río Balsas, en el mismo estado y en el de Puebla. Lo anterior también hace probable su presencia en Michoacán ya que parte de este se incluye en la cuenca del río Balsas.

\section{Hábitat y conservación}

La población silvestre de Cyatlea fulva que aquí se menciona tiene una distribución restringida, ya que es única en la sierra, se desarrolla en sitios sombreados en el fondo de una cañada, está compuesta por poco más de 60 individuos de diferentes edades, llegando a medir hasta 4 o $5 \mathrm{~m}$ de altura y se encuentra ubicada en la ladera noreste de la sierra, en La Cañada Río Frío, en las coordenadas $100^{\circ} 20^{\prime} 48.9^{\prime \prime}$ longitud O y $18^{\circ} 53$ '.53.8" latitud N, a 1900 msnm (figura 3).

Dicha cañada tiene una extensión aproximada de $700 \mathrm{~m}$ de largo y en el fondo corre un arroyo de flujo permanente cuyo nivel de agua llega a subir más de un metro en época de lluvias; en la zona hay una precipitación pluvial aproximada de $1375 \mathrm{~mm}$ en promedio anual, el clima es semicálido subhúmedo y se presenta un relieve accidentado con pendientes mayores a $60^{\circ}$ y cañadas que protegen a la vege- tación de la insolación y de los fuertes vientos. Algunos árboles del fondo de la cañada llegan a medir más de $20 \mathrm{~m}$ de altura y tener diámetros de alrededor de $110 \mathrm{~cm}$ a la altura del pecho. La vegetación donde se encontró el taxón corresponde a un bosque de galería con elementos característicos de bosque mesófilo de montaña, entre los cuales destacan especies como Matudea trinervia Lundell, Styrax ramirezii Greenm., Clusia salvinii Donn. Sm. y Clethra mexicana DC. La vegetación que rodea la cañada es un bosque de Pinus-Quercus, en la que dominan $P$. oocarpa Schiede ex Schltdl. y $Q$. urbanii Trel., con algunos elementos de Arbutus xalapensis Kunt y Clethra mexicana DC. Challenger (1998) menciona que los helechos arborescentes no existen en ningún otro tipo de vegetación primaria que no sea el bosque mesófilo de montaña, aunque se pueden encontrar en relictos o en ecotonos entre éste tipo de vegetación y la selva húmeda. Especies como $C$. costaricensis (Mett. Ex Kuhn) Domin, C. myosuroides (Liebm.) Domin, $C$. microdonta (Desv.) Domin y algunas otras de manera marginal se encuentran en bosques tropicales subcaducifolios o perenifolios a altitudes incluso cercanas al nivel del mar (Moran, 1995; Luna et al., 2001; Véliz y Vargas, 2006).

C. fulva es conocida localmente por los lugareños como "palma" y está ubicada en México en la categoría de “protección especial”, según la Norma Oficial Mexicana (Semarnat, 2002) además, está incluida en el apéndice dos de CITES (2007). En otros estados en los que se distribuye esta especie, se tienen reportes del uso del maquique para la elaboración de artesanías (Eleuterio y Pérez-Salicrup, 2006). En la Sierra de Nanchititla no se tiene ningún registro de su explotación artesanal, probablemente debido a que casi no se desarrolla maquique en los tallos de las plantas.

A pesar de ser Área Natural Protegida, el lugar en donde se encontró la especie está amenazado por 


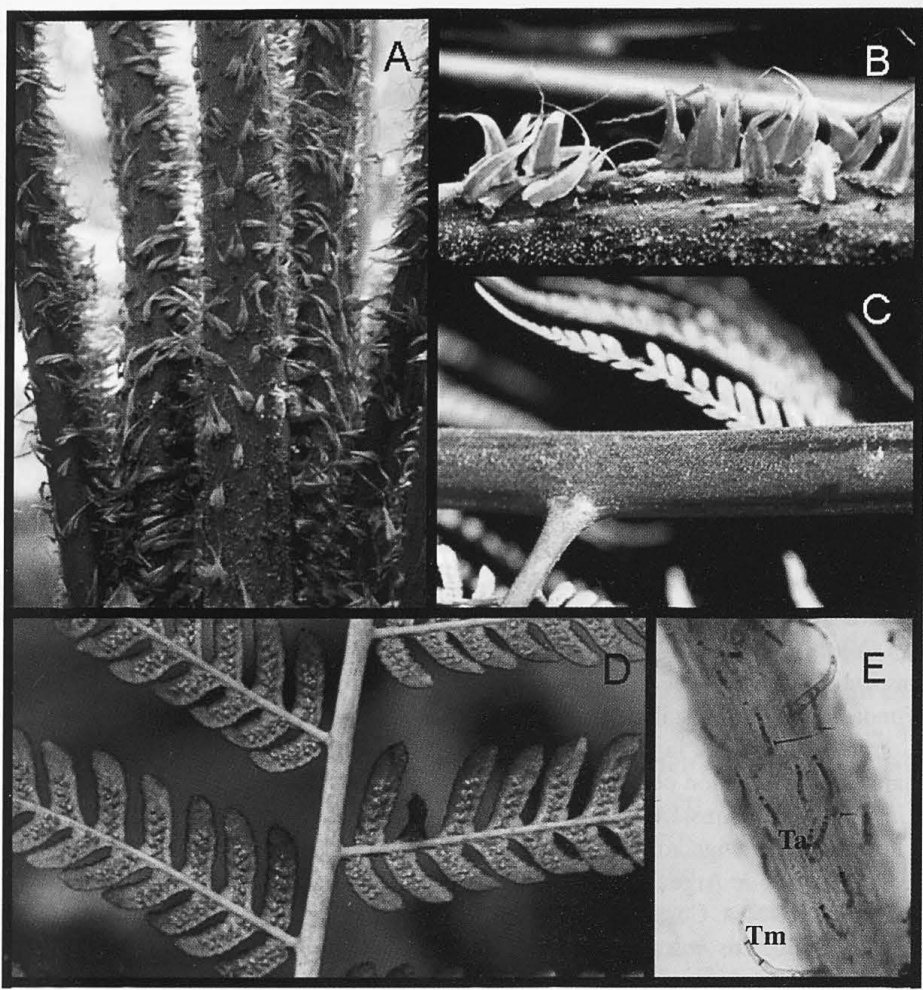

Figura 2. A, base de los pecíolos de Cyatlea fulva. B, escamas concoloras. C, caspilla parda en raquis. D, soros inframendios con indusio globoso y $\mathrm{E}$, cóstulas con tricomas adpresos (Ta) y multicelulares (Tm).

explotación forestal, agricultura y ganadería, por lo que es importante implementar medidas de protección y vigilancia que podrían ayudar a conservar y probablemente a incrementar el tamaño de dicha población, a la vez que se deben de desarrollar estrategias de manejo y monitoreo que permitan incrementar las probabilidades de su existencia. En este sentido, investigadores de la Universidad Autónoma del Estado de México están llevando a cabo estudios autoecológicos para hacer una evaluación mas precisa de la población. Es importante tener presente que para que estas plantas completen exitosamente su ciclo de vida, se requieren condiciones muy especiales, principalmente de humedad y luz, ya que primero se establecen y germinan las esporas, pasan por la fase del gametofito, la cual es muy delicada y ces se produce un esporofito, el cual va a madurar como la planta adulta que comúnmente se conoce, por lo que la más ligera modificación de su hábitat alteraría seriamente la población y en consecuencia el equilibrio del ecosistema. Es importante considerar que son plantas muy delicadas y de crecimiento muy lento, Riba (1978) ha calculado que en condiciones naturales, un helecho arborescente puede crecer entre 10 y $12 \mathrm{~m}$ de altura en aproximadamente 50 a 60 años.

El material recolectado (C. Zepeda, 30 de noviembre de 2007, No. 1620), se depositó en el Herbario de la Facultad de Ciencias de la Universidad Autónoma del Estado de México (HUAEM) $y$ en el Herbario Nacional de México (MEXU).

\section{Agradecimientos}

C. Zepeda y O. Monroy-Vilchis agradecen al pueblo mexicano por financiar este estudio a través de la Universidad Autónoma del Estado de México con los proyectos 2188/2005, 2356/2006, así como al Programa de Mejoramiento del Profesorado PROMEP-SEP (103.5/04/1304). La fundación Terra-Natura otorgó financiamiento a través del proyecto 2330/2006. A la CEPANAF por facilitarnos el acceso al Parque Sierra Nanchititla. Un agradecimiento especial a

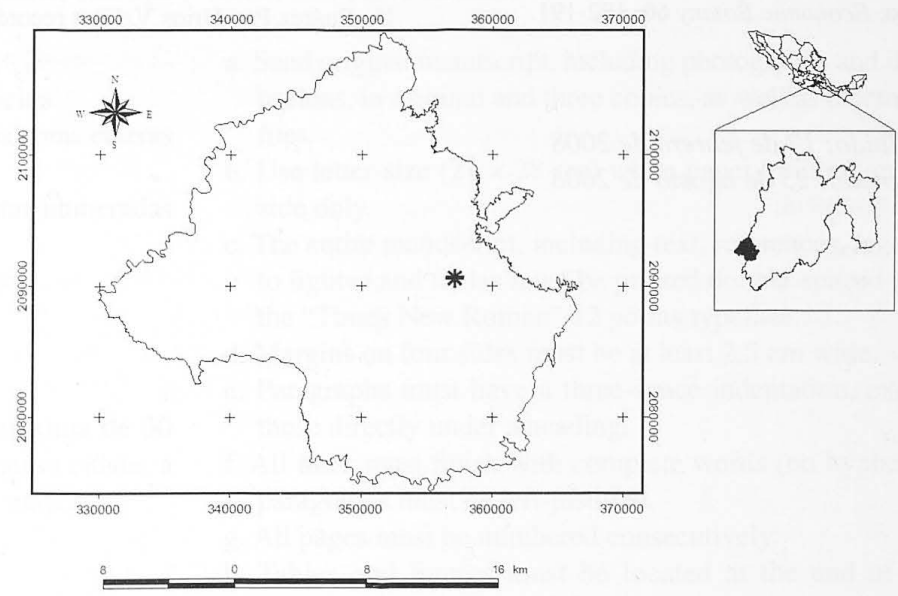

Figura 3. Mapa de localización de la Sierra Nanchititla, Estado de México. 
la familia Santander Ocampo quienes nos ofrecieron información de manera desinteresada, así como a los alumnos de la Estación Biológica Sierra Nanchititla y del Herbario de la Facultad de Ciencias de la UAEMéx por su participación siempre entusiasta. Agradecemos a Marco A. Romero Romero y Daniel Osorio González su apoyo logístico. La primera autora agradece a Javier Manjarrez el apoyo logístico. Agradecemos a los revisores anónimos cuyas observaciones enriquecieron este documento.

\section{Literatura citada}

Aguilera H. J. 1993. Los parques naturales del estado de México. CEPANAF. Estado de México. 121 p.

Anónimo. 1997. Programa de manejo del parque Sierra de Nanchititla. Secretaría de Ecología. Gobierno del Estado de México. Toluca, México. 110 p.

Challenger A. 1998. Utilización y conservación de los ecosistemas terrestres de México. Pasado, presente y futuro. Comisión Nacional para el Conocimiento y Uso de la Biodiversidad, Instituto de Biología, UNAM y Agrupación Sierra Madre, S. C. México.

CITES. 2007. Apéndices I, II y III. Consulta en línea el 10 de enero de 2008. http://www.cites.org/eng/app./e-appendices.pdf.

Eleuterio A. A. y Pérez-Salicrup D. 2006. Management of tree ferns (Cyathea spp.) for handicraft in Cuetzalan, Mexico. Economic Botany 60: 182-191.
Fernández-Nava R., Rodríguez C., Arreguín L. M. y Rodríguez A. 1998. Listado florístico de la cuenca del río Balsas, México. Polibotánica 9: 1-151.

Judd W. S., Campbell C. S., Kellog E. A., Stevens P. F. y Donoghue M. J. 2007. Plant Systematics. A phylogenetic approach. 3 a Ed. Sinauer Associates, Inc. Massachussets, USA. 611 p.

Lorea-Hernández F. y Velázquez-Montes E. 1998. Pteridofitas. Lista de los taxa y su distribución geográfica en la entidad. En: Diego-Pérez, N. y Fonseca R.M. Eds. Estudios Florísticos en Guerrero No. 9. Prensas de la Facultad de Ciencias, UNAM. México. 83 p.

Luna, I., Velázquez A. y Velázquez E. 2001. México. En: Kappelle, M. y Brown A.D. Eds. Bosques Nublados del Neotrópico, pp. 183-229, 1ª. Ed. Instituto Nacional de Biodivesidad, Fundación Agroforestal de Argentina, Comité Holandés de la Unión Mundial para la Conservación de la Naturaleza, Universidad de Amsterdam y el Laboratorio de Investigaciones Ecológicas de las Yungas de Argentina. Santo Domingo de Heredia, Costa Rica.

Matuda E. 1956. Los helechos del Estado de México. Gobierno del Estado de México. Dirección de Agricultura y Ganadería. Toluca, México. 72 p.

Mickel J.T. y Smith A.R. 2004. The Pteridophytes of Mexico. Memoirs of the New York Botanical Garden 88: 1- 1054.

Monroy-Vilchis O; Hernández-Gallegos O y Rodríguez-Romero F. 2005. Heloderma horridum horridum. Unusual habitat. Herpetological Review. 36: 450

Monroy-Vilchis O., Sánchez O., Aguilera U., Suárez P. y Urios V. First record of
Panthera onca in the State of Mexico. The Southwestern Naturalist. (En prensa).

Moran R.C. 1995. Cyatheaceae. En: Moran R.C. y Riba R. Eds. Flora Mesoamericana: Psilotaceae a Salviniaceae., pp 88-104, Universidad Nacional Autónoma de México. D.F. México.

Riba R. 1978. Los helechos arborescentes y el "maquique". INIREB Informa 25 : 1-4.

Sánchez O., Ramírez-Pulido J., Aguilera U. y Monroy-Vilchis O. 2002. Felid records from the State of Mexico, Mexico. Mammalia 66: 289-294.

Semarnat [Secretaría del Medio Ambiente y Recursos Naturales]. 2002. Norma Oficial Mexicana NOM-059-ECOL2001, Protección ambiental-Especies nativas de México de flora y fauna silvestres-Categorías de riesgo y especificaciones para su inclusión, exclusión o cambio-Lista de especies en riesgo. Diario Oficial de la Federación. 2a Sección, 6 de marzo de 2002.

Smith A. R., Pryer K. M., Schuettpelz E., Korall P., Schneider H. y Wolf P. G. 2006. A classification for extant ferns. Taxon 55: 705-731.

Tejero-Díez J. D. y Arreguín-Sánchez M. L. 2004. Lista con anotaciones de los pteridófitos del Estado de México, México. Acta Botánica Mexicana 69: 1-82.

Véliz, M. y Vargas J. 2006. Helechos arborescentes de Guatemala. Distribución, diversidad, usos y manejo. $1^{\text {a. }}$. Ed. Fondo Nacional para la Conservación de la Naturaleza, Organización Nacional para la Conservación y el Ambiente y Universidad de San Carlos de Guatemala. Guatemala, Guatemala. 94 p. 